\title{
Working Memory, Processing Speed, and Executive Memory Contributions to Computer-Assisted Second Language Learning
}

\author{
Keith E. Nelson \\ Pennsylvania State University, United States \\ Aran Barlieb \\ Pennsylvania State University, United States \\ Kiren Khan \\ Pennsylvania State University, United States \\ Elisabeth M. Vance Trup \\ Pennsylvania State University, United States \\ Mikael Heimann \\ Linkoping University, Sweden \\ Tomas Tjus \\ Gothenburg University, Sweden \\ Mary Rudner \\ Linkoping University, Sweden \\ Jerker Ronnberg \\ Linkoping University, Sweden
}

\begin{abstract}
How individual differences in information processing affect second language (L2) learning has been unclear in prior research. Adults lacking prior skill in Swedish were pretested for working memory, processing speed, and executive memory capacity. Participants then received 6 computer-based instructional sessions with pictorial animations of Swedish sentences, with a built-in experimental contrast between some lessons at high and some at low rates of presentation. The faster rate carried greater processing demands for the learners. Higher levels of Swedish performance during Instructional sessions were associated with higher working memory levels, as expected from widely-used models of working memory (e.g., Baddeley \& Hitch, 1994). In contrast, results at demanding long-term retrieval on a posttest were more complex and revealed several dynamic relationships between processing speed, working memory, and Swedish language learning. Learners with low rather than high working memory showed higher L2 skills at long-term testing when instructional lessons had employed fast animations. This first-time demonstration that prior cognitive profiles strongly influence learners' progress in second language requires refinements in existing theories.
\end{abstract}


Further, the results hold certain implications for tailoring second language teaching on-line or in other technology-based instruction to learner profiles on abilities in working memory, processing speed, and executive memory.

Keywords: Educational technology; Software; Designing learner-sensitive procedures; Computer-assisted learning; Second language acquisition; Dynamic systems

\section{Introduction}

Adults and adolescents frequently seek to acquire significant skills in a second language (L2). Yet progress toward that goal is highly variable, regardless of whether the context of learning is in classrooms or informal learning after immigration to a new country. Surprisingly, despite many theoretical models that place information processing at the heart of language learning, prior research has not examined detailed processing profiles of individuals as possible prospective predictors of their rates of progress once they begin learning a second language. The current study sought to fill that research gap. Specifically, the study tests how initial individual differences in information processing affect later progress in second language acquisition in Swedish under computer-assisted instruction.

In the related field of adults' performance in their well-established first language (L1), a rich literature demonstrates that both oral language performance and written language performance are influenced by a variety of information-processing differences. Working memory, processing speed, and executive memory (or "executive attention") have all been shown to be important components that contribute to speed and efficiency of completing reading tasks and oral language comprehension and verbal memory tasks. A number of theoretical models that strive to account for these empirical findings share assumptions that better adult L1 performance is supported by better information processing capacities. Models differ in whether they conceptualize available information processing capacities in terms of an overall pool of resources (e.g. Cowan, 2005; Just \& Carpenter, 1992; Miyake, 1994) or in terms of separable components/buffers that work together but have distinct roles such as a "phonological loop," a multimodal episodic/comparison buffer, and a component of executive control or executive memory/attention (Baddeley, 2000; Baddeley \& Hitch, 1994; Gathercole \& Baddeley, 1993; Gupta \& MacWhinney,1997; MacWhinney, 2008; Repovs \& Baddeley, 2006; Rudner \& Ronnberg 2008).

Another important set of observations for adults' use of their well-practiced, well-consolidated first language cover what happens when dynamic conditions in real time are varied. Under highly familiar and undemanding conditions, many steps in processing incoming language may be handled mostly in an "implicit" manner--with high efficiency and without much conscious effort. In contrast, when language comprehension becomes complicated by a noisy or distracting environment or by complex linkages between prior and current elements in a narrative, more information processing resources may have to be dedicated to relatively slow and explicit analyses involving more extensive retrieval cycles and more participation of executive memory/attention components (Rudner \& Ronnberg, 2008; Ronnberg, Rudner, Foo, \& Lunner, 2008). Similar complexities in relation to employing information-processing resources in varied tasks and contexts have been noted by many other theorists (Turner \& Engle, 1989; Just \& Carpenter, 1992; 
Miyake \& Friedman, 1994). Hung and colleagues (Hung, 2006, 2009, 2011; Jonassen \& Hung, 2008) address the need for educational technology research that is both theory-based and very explicit about how variations in problem-based learning designs do or do not engage and support learners. Another example from educational technology research along these lines is that in multiuser virtual learning environments the "cognitive load" placed upon learners has been analyzed, and then manipulated for users overall by varying mode (e.g. speech, text) of certain inputs (Erlandson, Nelson, \& Savenye, 2010). In the present study and in future research it will be interesting to see how cognitive differences between individuals in their information processing intersect with the organization and pace of content presented in varied educational technology teaching situations.

All of the literature on use by adults of their well-consolidated, well-practiced first language (L1) provides clues to information-processing components that might behave in similar ways when explored in early stages of second language (L2) acquisition. However, which components contribute and in what manner to individual differences in L2 progress remains to be determined. In the current study researchers chose to track $L 2$ acquisition within an innovative technology context because the software employed allows precise control of how often each language structure is encountered along with the specific patterns of presentation.

\section{Expectations of Impacts on L2 Learning of Individual Differences in Information Processing}

The literature to date has provided some studies of adults that show clear cut association between individuals' higher measures of information processing capacities and concurrent higher performance levels in L2, but most of these have looked only at working memory and not all such studies report a significant correlation of working memory and L2 proficiency (Miyake \& Shaw, 1999). Further, unlike the present study, these studies have not been prospective: they have not measured information processing before learning encounters in L2 occur. The present study not only takes that step using multiple processing measures but also examines experimental contrasts in presentation speeds of learning materials.

The context for the present study will be adults who have individual sessions for computerassisted second language learning. However, findings on how learners' cognitive profiles contribute to learner progress are expected to have implications for innovations in design of learning procedures in a broad variety of multi-user and individual-learner contexts where educational technology is employed with adults or children.

\section{Design of the Present Study}

Prior literature has not examined in a prospective research design how much processing speed, executive memory, and working memory contribute to the earliest stages of second language (L2) learning. Therefore, the present study is designed to fill that gap. The researchers look at how these factors as well as the factor of animation presentation speed affect Swedish language acquisition by English-speaking adults from the very first words and sentences they encounter through educational software. The central hypothesis of this study is that individual differences in 
these four processes will contribute both to differences in initial learning tested during Swedish instructional sessions and to differences in long-term retrieval of Swedish language at the posttest at the end of the study.

\section{Methodology}

\section{Participants}

Participants were 25 college students in U.S., ages 19 to 30 (mean $=21.1$ ), recruited through invitations to join the study. They were required to have no previous exposure to the Swedish language. They all completed a Session 1 Pretesting for information processing skills, Instruction in Swedish with educational software in Sessions 2 to 7, and Posttesting on Swedish at Session 8.

\section{Measures}

\section{Pretest Measures of Information Processing}

Processing speed (IV-1): Processing speed refers to the speed at which a broad range of cognitive information-processing steps can be completed by an individual. A rapid color naming subtest asked participants to name the color of each in a series of colored squares as rapidly as possible. The rapid object naming subtest asked the participant to recite the name of the object in a series of pictured objects as rapidly as possible. Correct answers were totaled.

Working memory (3 measures): Working memory is considered to be a working space of limited capacity in which needed information is temporarily stored and current steps in thinking are carried out with high attentional focus. The digit span task (IV-2) asked participants to successfully repeat increasingly long series of numbers (Gathercole, Service, Hitch, Adams \& Martin, 1999). The word span task (IV-3) asked participants to successfully repeat increasingly long series of words. Nonword repetition, often used as a measure of a presumed "phonological loop" component of working memory, required the participant to listen to strings of phonologically acceptable nonwords that have no lexical meaning and repeat these.

Executive memory: Executive memory/attention requires the coordination of multiple goals and operations along with inhibition of any competing distractors. The Operation-Span memory task (IV-4) measured participants' executive memory (Turner \& Engle, 1989). Participants identified a series of mathematical equations as either correct or incorrect while memorizing English words that showed up in between each equation. After a series of equations that varied in terms of set size from 2 to 6 , the words from that particular set were to be recalled. Equal points were given for correctly answering the mathematical equation and recalling the words for a maximum score of 60 . 


\section{Measures of Progress in Swedish During Instruction}

Average correct performance on the last 3, most complex, lessons was calculated for each participant (DV-1). Each item on these tests during Omega-Is software (Heimann, Lundalv, Tjus, \& Nelson, 2006) instruction required the learner to make a Swedish text sentence that matched in meaning with an animation of a complex event (e.g., The singing fox sails across the lake and helps the bear.).

\section{Measures of Swedish Performance at Post-test}

Large Long-term Test of Swedish: The maximum total score on this largest post-test of Swedish understanding was 53 (DV-2). Items included 15 single words and 38 sentences or phrases. Each item presented Swedish text and asked the participant for an English text translation.

Recalling from fast-presentation lessons compared with slow-presentation lessons. Each participant saw 20 items ( 3 single words, 3 phrases, and 14 sentences) from lessons viewed during instruction in the fast speed for animation $(3.3 \mathrm{sec})$ and 20 from lessons viewed in the slow $(6 \mathrm{sec})$ speed. Points were awarded for number of correct English translations from the Swedish text. (DV-3).

Verbal Fluency Longterm Retrieval Test. Each participant had 1 minute to verbally recall as many Swedish words as they could. Each attempt to recall a word was judged from audiotape by a native Swedish speaker on this scale: 4/native Swedish pronunciation, 3/good, 2/fair, 1/barely recognizable, and 0/not recognizable. Total points earned were tallied (DV-4).

\section{Procedures}

During session 1, Pre-test, participants completed the processing speed, working memory, and executive memory pretest measures.

The earliest 3 of the 6 instructional sessions (sessions 2 to 4) with Omega-is software built the learners' confidence and established an initial foundation of Swedish words and simple Swedish sentences. Then complexity increased. Longer, more complex sentences were created in the last 3 instructional sessions (sessions 5 to 7), with up to 14-word complex sentences. An example is The blond-haired girl kicked the soccer ball over the net and into the helicopter. Following the creation of a phrase/sentence in Swedish text, the voice would repeat the entire phrase/sentence following which an animation would display the exact meaning of that phrase/sentence. After the instructed number of phrase/sentences were created within a session, tests showed the animation first and then the correct phrase/sentence in text was to be created by the learner from the bank of possible Swedish words.

Presentation rates were systematically varied: half the lessons used animations at fast rates and half at slow rates. 
Session 8, Post-test, occurred at least 48 hours after the final (sixth) Swedish instructional session, and drew upon earlier lesson material as well as from the first 5 instructional sessions. Accordingly, the long-term retrieval interval between initial learning of particular L2 Swedish content and session 8 post-testing was variable depending on when a test structure had last been seen in instruction, falling between 2 and 14 days.

\section{Results}

As hypothesized, individual differences at pretest on multiple information processing measures were predictive of differences in acquisition of Swedish as a second language in an educational technology context. All such relationships relating information processing factors (IVs) to measures of progress in Swedish (DVs) were examined through Analyses of Variance (ANOVAS).

\section{Performance on Swedish Lessons during Computer-Assisted Instructional Sessions}

Average performance on sentences for the final 3 lessons was significantly higher for learners with High or Medium Working Memory/Digit-Span (IV-3) abilities at pretest as compared with those with Low Working Memory/Digit-Span abilities at pretest, $F(2,21)=5.02, p<.025$, partial eta square $=.302$, very large effect size. This result, shown in Figure 1 , is one confirmation of the key hypothesis of the study.

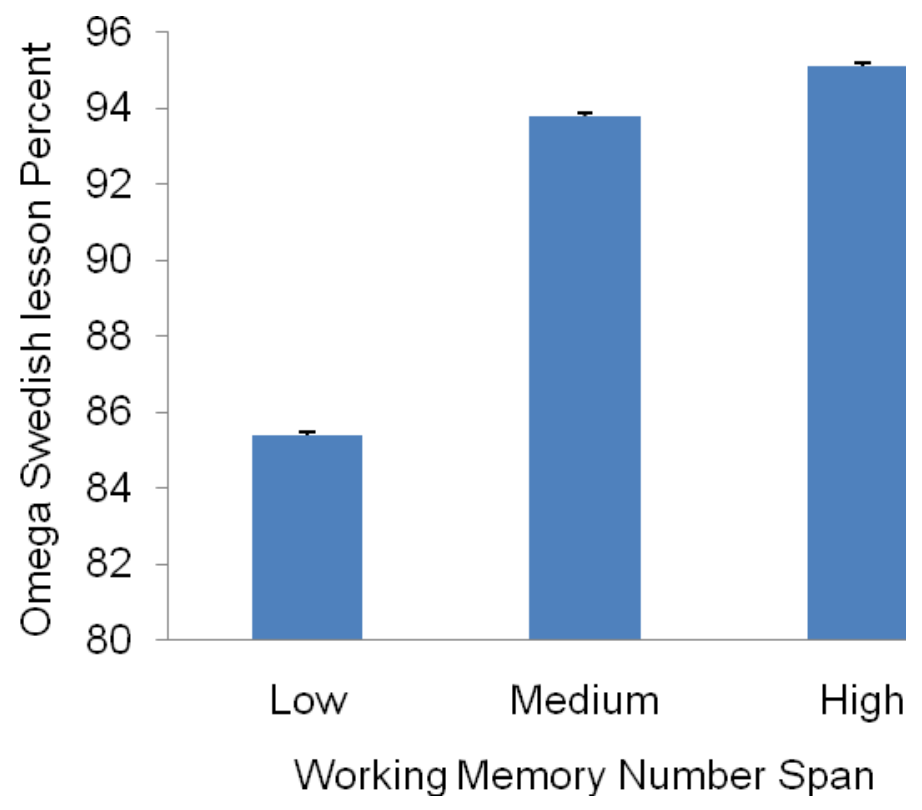

Figure 1. Mean Percent Correct (with standard error) on the Last Three Omega Swedish Instructional Sessions at Each of Three Working Memory Levels (low, medium, or high) based on Performance on a Digit Span Task 


\section{Verbal Fluency in Swedish at Longterm Retrieval at Post-test}

Total recognizable Swedish words produced in one minute in the session 8 post-test were recorded and then scored from audio by a Native Swedish speaker. Highest phonological accuracy was represented by words scored as "4" for Native-like pronunciation. As Figure 2 shows, higher numbers of these Native-like words at longterm retrieval were associated with higher rather than lower information-processing abilities as measured by a composite of Nonword Repetition (IV-5, often used as a "phonological loop" measure) and Operation-Span (IV-6, measuring Executive Memory/Attention), $F(1,21)=4.89, p<.04$, partial eta squared $=.188$, very large effect size.

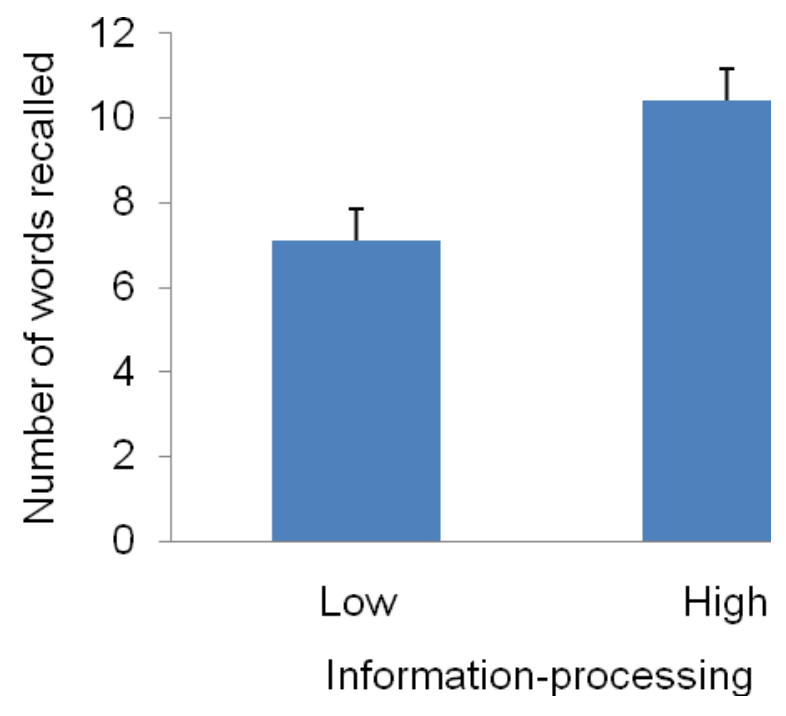

Figure 2. Mean Performance (with standard error bars) on Oral Recall of Swedish Words for Participants with High versus Low Information-processing Abilities as Measured by a Composite of Nonword Repetition and Operation-span

\section{Performance in Swedish Overall Based Upon Longterm Retrieval at Post-test}

Another measure of Swedish performance at posttest was a large sample of Swedish items encountered during the instructional sessions. Learner performance, here translating Swedish text into English text, again varied by pretest cognitive abilities. In this instance, learner performance was an interaction shown in Table 1 between Working Memory/Digit-Span (IV-3) and Processing Speed/Object Naming (IV-2), $F(2,18)=4.50, p<.03$, partial eta squared $=.333$, very large effect size. Learners with Low Processing Speed showed complex variation according to levels of Working Memory, with best performance for those with Medium Working Memory. In contrast, Learners with High Processing Speed showed increasing performance as levels of Working Memory declined. As part of this interaction, it is thus apparent that the two subgroups with the highest Swedish performance based upon longterm retrieval were participants with Low 
Processing Speed and Medium Working Memory or High Processing Speed and Low Working Memory, whereas the very lowest Swedish performance was for participants with a combination of Low Processing Speed and Low Working Memory.

Table 1. Mean (with standard deviation) Performance on a Large Swedish Test of Long-term Retrieval as Related to Working Memory and Processing Speed

\begin{tabular}{|c|c|c|c|}
\hline & $\begin{array}{c}\text { Low Working } \\
\text { Memory }\end{array}$ & $\begin{array}{c}\text { Medium Working } \\
\text { Memory }\end{array}$ & $\begin{array}{c}\text { High Working } \\
\text { Memory }\end{array}$ \\
\hline High processing speed & $32.3(3.21)$ & $28.8(8.04)$ & $24.0(3.00)$ \\
\hline Low Processing Speed & $22.0(7.57)$ & $34.5(4.12)$ & $28.6(4.04)$ \\
\hline
\end{tabular}

*Working Memory from the Digit Span task was divided into groups with low, medium, and high performance. Processing speed, as measured by performance on the Rapid Object Naming measure was divided between low and high processing speed.

These results contrast with the two findings above and they suggest that for longterm retrieval of new Swedish text understanding there are dynamic interactions of information-processing components that contributed during Instruction to the deepest and fullest levels of processing achieved.

\section{Performance in Swedish Based Upon Longterm Retrieval at Posttest from Software Lessons Presented at High versus Low Presentation Rates}

Performance was first examined here for a set of 20 sentences that had been presented at Fast Presentation Rates during the Instructional sessions. The dependent variable here was accuracy at Post-test of translating these Swedish sentences (L2) into English (L1). Learner performance was predicted significantly both by Working Memory/Word-Span (IV-4), $F(2,18)=5.60, p<.03$, partial eta squared $=.237$, very large effect size, and by Processing Speed/Color-Naming (IV-1), $F(2,18)=$ $4.80, p<.02$, partial eta squared $=.348$, very large effect size. Swedish longterm performance was higher for lower cognitive levels at pretest for Working Memory (see Figure 3).

Similarly, Figure 4 demonstrates that Swedish performance was higher for learners with Low Processing Speed than for those with either Medium or High levels of Processing Speed. These findings on Swedish performance at posttest, where longterm retrieval was required for translating Swedish text into English text, again implicate interesting dynamic interactions of information-processing components during instruction contributing to deep processing and highly successful encoding of new L2 knowledge into longterm memory. 


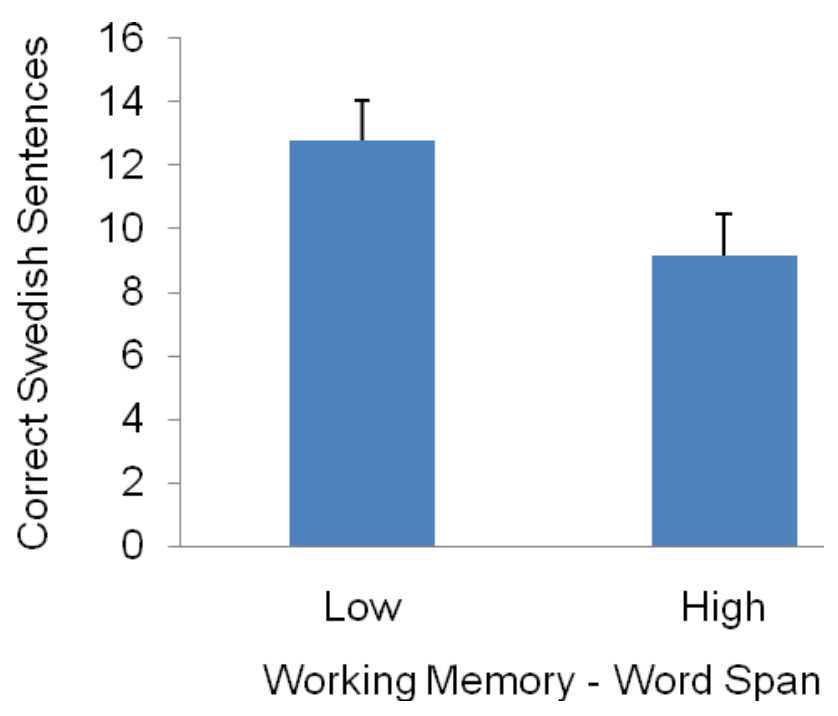

Figure 3. Mean Performance (with standard error bars) for Performance on Written Recall of Sentences Previously Presented at Fast-speeds during the Lesson 8 Long-term Retrieval Post-test. (Performance on Swedish for participants with high versus low scores on Working Memory as measured by Word Span).

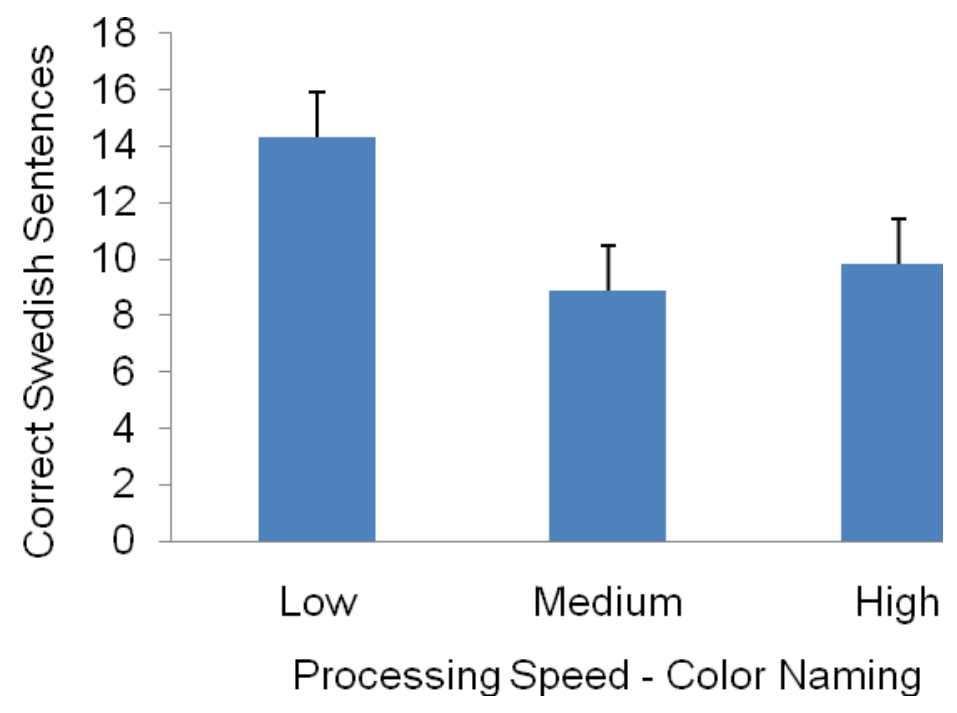

Figure 4. Mean Performance (with standard error bars) for Performance on Written Recall of Sentences Previously Presented at Fast-speeds during the Session 8 Long-term Retrieval Post-test (Performance on Swedish for participants with low, medium, or high processing speeds based on Color Naming)

For a set of sentences that had been presented at Slow Presentation Rates for the animations, performance on the long-term testing showed no systematic relation to any of the pretest 
measures of Information Processing. It would seem that learners of all levels of information processing abilities were able to establish similarly successful longterm memory representations of the Swedish material presented during Instruction if material was presented at the slow (twice the fast rate) rate.

\section{Conclusion}

Multiple findings confirm the central hypothesis of this study that individual differences in multiple components of information-processing prior to L2/Swedish software instruction would contribute both to differences in initial learning during instructional sessions and to differences in long-term retrieval of Swedish language at the end of the study. This is the first empirical demonstration of such complex information-processing profiles predicting subsequent individual differences in gains in L2 (second language) skills.

\section{L2 Performance During Instruction}

Working memory did predict Swedish language learning. The first such finding is that performance during the final three instructional lessons was directly related to level of pre-instruction working memory as measured by the very commonly-employed digit span task. The high and medium working memory groups performed significantly better on the Omega lesson tests than did the low working memory group

\section{L2 Performance Based Upon Long-term Retrieval}

Certain pretest, pre-instruction measures of information processing also positively predicted verbal fluency as indicated by long-term retrieval at the post-test of Spoken Swedish words. In this case we see that the only result anywhere in this study that was associated with a key measure of the "phonological loop", nonword repetition (Gathercole \& Baddely, 1993; Baddeley \& Hitch, 1994; Gathercole et al., 1999). Fluency, in terms of more L2 words produced at Native-like pronunciation levels, was significantly related to higher scores on a measure that combined nonword repetition with a measure of Executive memory (Operation-span).

Working Memory together with Processing Speed affected performance in an interactive way on this study's largest test of post-instructional, Long-term Swedish Retrieval. Being low in either processing speed (speed of naming objects) or working memory (span of remembered digits) while also being medium-to-high in one of these components led to more Swedish L2 learning than being high in both.

On another long-term measure, performance levels on the posttest of sentences specifically seen at the Fast Presentation Rate during instruction were predicted by working memory as measured by the word span task and processing speed as measured by the rapid color naming task. It is interesting that for encoding into longterm memory during Instruction with fast animations, and then delayed, longterm retrieval at post-test, it was a verbal span measure of working memory 
that was predictive. Performance on the posttest was higher for those with lower working memory scores than for those with high working memory scores. Scores were also higher for those with low processing speed as compared with medium or high processing speed. As we will see, such results favoring individuals with lower information-processing capacities on some measures of $L 2$ learning pose difficulties for many but not all theoretical models of memory and learning.

Some of the dynamic interactions of information processing components appear to be similar to parts of Newport's (1990) "Less is More" Hypothesis. This hypothesis states that as an individual's age and development increases, certain aspects of their language learning potential declines because, paradoxically, cognitive abilities increase. Children at ages 1.5 to 3 years of age may more easily perceive and store component sentence parts rather than complex wholes like adults. Consequently, under the actual dynamic conditions of encountering new syntactic challenges, young children are better at isolating parts required for recombination. Lower information processing capacity for some adults, such as that in young children, could then lead to better progress in the earliest stages of syntax learning.

This hypothesis may fit well with other dynamic systems accounts of why in this study at longterm retrieval lower information processing capacities sometimes were associated with higher Swedish retention and performance. Dynamic interaction of information processing components, as discussed by many authors (Demetriou et al., 2002; Elman et al., 1996; Gupta \& MacWhinney, 1997; Nelson, 1989, 2000, 2006; Nelson et al., 2001; Nelson \& Arkenberg, 2008; Peltzer -Karpf \& Zangl, 2001; Roberts \& Pennington, 1996; Thelen \& Smith, 1996), appears to be a plausible explanation of why those with high processing speed and low working memory performed better than those with high processing speed and high working memory on the largest Overall Swedish posttest. Those with lower working memory may have been more capable of learning Swedish component parts during Instruction to a deep level of longterm memory encoding, and could therefore demonstrate longterm retention at posttest.

Likewise, this same dynamic account of some paradoxically higher levels of encoding during Instruction depending upon not-too-high processing speed and not-too-high working memory could explain why performance on the posttest of sentences presented at the fast pace during Instruction was better for participants with low working memory or with low processing speed. When each part of a sentence is processed a bit slower and in the presence of a smaller "span" of items simultaneously in working memory, then each part may receive fuller, deeper encoding into longterm memory. For the tests during Instructional sessions that relied upon relatively shorterterm memory, these dynamic interactions were not apparent, but were visible only when Swedish L2 performance was examined at the longer-term post-testing.

\section{Comparison of Multiple Information-Processing Models Relevant to Learning in Educational Technology Contexts}

In comparison with the other models of memory and information processing considered here, Nelson and colleagues have emphasized more strongly how much parallel processing (Calvin, 1990) contributes to successful learning episodes in language (L2 and L1) and how much variation occurs in the dynamic convergence patterns of multiple abstraction, working memory, retrieval, 
attention, and executive function processes when individuals encounter contrasting contextual conditions (Nelson, 1991, 2000, 2006; Nelson et al., 2001; Nelson et al., 2004; Nelson \& Arkenberg, 2008). Relatedly, as learners shift from early to late stages of acquisition of particular syntactic structures (e.g., passives, future tenses), there are shifts too in required information-processing steps (Nelson, 1991; Nelson et al., 2004; Nelson et al., 2001). The complexity of understanding the particular mixes of conditions/processes determining when individual learners will make the most versus least progress in first language or second language learning (Gillum, Camarata, Nelson, \& Camarata, 2003; Nelson, 2006; Nelson et al., 2004) is emphasized through the label of Dynamic Tricky Mix Theory. Similarly, the Rapid Automatic Binding of Phonology (RAMBPHO) model of language comprehension stresses that when contextual conditions (e.g., increased noise) shift, there will be shifts in how multiple information-processing components pattern together to support language understanding (Ronnberg et al., 2008; Rudner \& Ronnberg, 2008). From both the RAMBPHO and Dynamic Systems theoretical frameworks, it makes excellent sense that different information-processing components and different measured capacity levels within these components will be the varied strongest predictors of measured learning for different probes into second language learning. Among important learning outcomes to consider are highly primed words and sentences during tests within learning sessions, longterm retrieval overall, longterm retrieval of sentences/animations presented during instruction at demanding high rates, and longterm retrieval orally of Swedish words with Native-like pronunciation.

\section{A Closer Look at Steps in Information Processing During Language Acquisition}

Performance during instructional lessons was predicted positively by one common measure of working memory capacity, the span (length) of digits that can be immediately recalled after presentation. Higher spans may have insured that more Swedish text would be in working memory when a meaningful animation followed. Further, since words and phrases making up the exploration/instructional sentence examples were repeated in different sentences, there was considerable potential for recent priming of these items, and higher working memory may have led to stronger priming effects. These lines of interpretation fit well with models proposed by Baddeley and colleagues (e.g. ,Baddeley, 2000; Baddeley,\& Hitch, 1994; Repovs \& Baddeley, 2006).

In the case of long-term retrieval of spoken Swedish words, it is important to note there was no retrieval testing during instructional sessions. Nevertheless, learners had an opportunity to carry out sufficient processing steps for acquiring meanings for oral Swedish. In every case where a Swedish phrase or sentence was employed in instruction, the oral Swedish was also produced by the computer software. Learners knew that they would soon be tested for their use of Swedish text, so would be expected to put a priority in first forming comparisons in some "episodic buffer" or "executive memory" space of text to animations to retrieved English L1 representations. Once these comparisons were ongoing, to proceed to comparisons also of the spoken Swedish would seem to depend on two essential processes. First, good phonological working memory of some kind would need to hold the trace of the oral Swedish. Secondly, especially good "episodic buffer" or "executive memory" (as measured in this study by a pretest Operation-span task) would help ensure that the shift to comparison of the oral Swedish to animations and/or retrieved L1 English proceeded smoothly. In line with this analysis, the present results show that learners higher rather 
than lower on a composite of phonological working memory (nonword repetition) and OperationSpan measures were able to freely recall at post-test more Swedish words with Native-like pronunciation. This result, like the finding above that higher digit span predicts L2 short-term performance during instruction, appears compatible with a wide range of models of oral language and/or reading/writing. These models include those variously emphasizing overall informationprocessing resources (Just \& Carpenter, 1992), executive attention/memory (Shallice, 2004), a multimodal buffer joined to phonological and visual buffers (Repovs \& Baddeley, 2006; Ronnberg et al., 2008; Rudner \& Ronnberg, 2008), and dynamic models that incorporate an executive/ comparison buffer joined to multiple parallel buffers (Nelson et al., 2004).

Consider, finally, more details of possible dynamic interactions of information processing components during L2 instruction with fast animation speeds provided by the technology. The different phrases that made up sentences were encountered multiple times in different particular sentences and so this set up the possibility that there could be long-term learning advantages of only partially "capturing" a text sentence into working memory space. This scenario is most likely for individual learners with relatively low processing speed (affecting how fast each text word enters working memory) or relatively low working memory word span (affecting how many words can be held at one time in working memory). For the sentence, The whale put the pizza on the gorilla's table, perhaps for these learners only The whale put the pizza would be resident in working memory when the fast animation of the whole sentence is encountered. Better, deeper encoding of meanings into long-term memory may then occur for these learners who have less than the full Swedish text in working memory because they can complete more processing steps for their shorter stored text string than can be completed by learners who have the full text string to try to encode as test/animation paired meanings. If this same pattern then occurs across many different fast-animation presentations, then over the full set of instructional opportunities this would account for the demonstrated better long-term retrieval by learners with low working memory or low processing speed. This result appears to be paradoxical and difficult to interpret from the Baddely and colleagues' models of working memory (Baddeley, 2000; Baddeley \& Hitch, 1994; Gathercole, Willis, Emslie, \& Baddeley, 1992; Repovs \& Baddeley, 2006). In contrast, this result fits more easily with dynamic systems and other highly interactive models (Just \& Carpenter, 1992; Nelson \& Arkenberg, 2008; Nelson et al 2001; Ronnberg et al., 2008; Rudner \& Ronnberg, 2008; Thelen \& Smith, 1996). At the same time, these latter models would profit from refinements to more specifically account for early to late stages of acquisition for both first language (L1) and second language (L2).

The present study employed computer-based individual instruction of adults, with experimental contrasts between slow and fast animation presentations, for a relatively small sample size of 25 subjects. Nevertheless, theoretically-predicted and conceptually important differences did reach significance, with very large effect sizes in each case. Information-processing profiles of beginning L2 learners as well as Presentation Speed of lessons do indeed help to account prospectively for differences in L2 acquisition. Future research could build upon these findings by investigating how particular syntactic and semantic structures can best be taught to individuals with varying information processing profiles, by using technology to experimentally vary other features of lesson presentation in addition to animation speeds, and by studying second language acquisition prospectively in contexts other than software used for 1-to-1 instruction. 
More broadly speaking, not only for language but also for science, math, literacy, problem-solving and many other domains of learning, the present approach raises possibilities for experimentally establishing "best fits" between lesson design and individuals' cognitive characteristics (Erlandson, Nelson, \& Savenye, 2010). It would seem likely that on-line distance uses of educational technology would be particularly helpful in arranging multiple ways of trying to fit individual learners' cognitive profiles to lessons that vary widely in their timing, structure, redundancy, and related parameters. The present results strongly indicate that learners' individual differences in working memory, processing speed, and executive memory are important in learning progress. What remains to be done is to find ways of adjusting how educational technology arranges learning opportunities so that the field discovers which learning designs and feedback patterns help which students significantly (Fiddler \& Knoll, 1995; Jonassen \& Hung, 2008). As just one example, what could be a too-fast pace of encounters with learning challenges for some learners could be shown to be a highly engaging and effective pace for other learners. Systematic research along these lines for all kinds of educational technology contexts could establish for multiple aspects of cognition much better ways of teaching to individuals. In turn, cycles of improvement along these lines for educational technology implementations might help overcome what some in the field have seen to be limited evidence for important contributions to learning of educational technology as it has been deployed in many classroom and distance-learning situations (e.g. Cradler, 2003; Hung, 2006a, 2006b; Lagrange, Artique, Laborde, \& Trouche, 2001).

\section{Acknowledgments}

This research was supported in part by grants from the Schreyer Honors College and the College of Liberal Arts at Penn State University.

\section{References}

Baddeley, A. (2000). The episodic buffer: A new component of working memory? Trends in Cognitive Science, 4, 593-596.

Baddeley, A., Gathercole, S., \& Papagno, C. (1998). The phonological loop as a language learning device. Psychological Review, 105(1), 158-173.

Baddeley, A. \& Hitch, G. (1994). Developments in the concept of working memory. Neuropsychology, 8(4), 485-493.

Calvin, W. (1990). The cerebral symphony. New York: Bantam.

Cradler, J. (2003). Technology's impact on teaching and learning. Learning and Leading with Technology, 30, 54-57.

Cowan, N. (2005). Working memory capacity. New York: Psychology Press.

Demetriou, A., Christou, C., Spanoudis, G., \& Platsidou, M. (2002). The development of mental processing: efficiency, working memory, and thinking. Hoboken: Wiley-Blackwell.

Elman, J. L., Bates, E. A., Johnson, M. H., Karmiloff-Smith, A., Parisi, D., \& Plunkett, K. (1996). Rethinking innateness: A connectionist perspective on development. Cambridge, MA: MIT Press. 
Erlandson, B. E., Nelson, B. C., \& Savenye, W. (2010). Collaboration modality, cognitive load, and science inquiry learning in virtual inquiry environments. Educational Technology Research \& Development, 58, 693-710.

Fiddler, M. B. \& Knoll, J. W. (1995). Problem-based learning in an adult liberal learning context: Learner adaptations and feedback. Continuing Higher Education Review, 59(1/2), 13-24.

Gathercole, S. \& Baddeley, S. (1989). Evaluation of the role of phonological STM in the development of vocabulary in children: a longitudinal study. Journal of Memory and Language, 28, 1-14.

Gathercole, S. \& Baddeley, A. (1993). Working Memory and Language. Hillsdale, NJ: Lawrence Erlbaum Associates.

Gathercole, S.E., Service, E., Hitch G.J., Adams, A. \& Martin, A.J. (1999) Phonological short term memory and vocabulary development: further evidence on the nature of the relationship. Applied Cognitive Psychology, 13, 65-77.

Gathercole, S. E., Willis, C., Emslie, H., \& Baddeley, A.D. (1992). Phonological memory and vocabulary development during the early school years: A longitudinal study. Developmental Psychology, 28, 887-898.

Gillum, H., Camarata, S., Nelson, K. E., \& Camarata, M. N. (2003). A comparison of naturalistic and analog treatment effects in children with expressive language disorder and poor preintervention imitation skills. Journal of Positive Behavioral Interventions, 5, 171-178.

Gupta, P. \& MacWhinney, B. (1997). Vocabulary acquisition and short-term memory: Computational and neural bases. Brain and Language, 59, 267-333.

Heimann, M., Lundalv, M., Tjus, T., \& Nelson, K. E. Omega-is: Omega interactive sentences software. Gothenburg, Sweden: Topic Dos Hb, and Warriors Mark, PA, United States: Super Impact Images Inc.

Hung, W. (2011). Theory to reality: a few issues in implementing problem-based learning. Educational Technology Research \& Development, 59, 529-552.

Hung, W. (2006). The 3C3R model: A conceptual framework for designing problems in PBL. Interdisciplinary Journal of Problem-based Learning, 1, 55-77.

Hung, W. (2009). The 9-step process for designing PBL problems: Application of the 3C3R model. Educational Research Review, 4(2), 118-141.

Jonassen, D. H. \& Hung, W. (2008). All problems are not equal: Implications for PBL. Interdisciplinary Journal of Problem-Based Learning, 2(2), 6-28.

Just, M. \& Carpenter, P. (1992). A capacity theory of comprehension: Individual differences in working memory. Psychological Review, 99, 122-149.

LaGrange, J. B., Artigue, M., Laborde, C., \& Trouche, L. (2001). A meta-study on IC technologies in education: Towards a multidimensional framework to tackle their integration. Paper presented at the Proceedings of the 25th Conference of the International Group for the Psychology of Mathematics Education. ERIC document: ED 466950. 
Lonigan, C. J., Wagner, R. K., Torgeson, J. K., \& Rashotte, C. A. (2002). Preschool comprehensive test of phonological processing. Florida State University.

MacWhinney, B. (2008). A unified model. Handbook of cognitive linguistics and second language acquisition (pp. 341-371). New York: Routledge.

Miyake, A. \& Shah, P. (Eds.). (1999). Models of working memory: Mechanisms of active maintenance and executive control. Cambridge: Cambridge University Press.

Nelson, K. E. (1991). On differentiated language-learning models and differentiated interventions. In N. A. Krasnegor, D. M. Rumbaugh, R. L. Schiefusbusch, \& M. Studdert-Kennedy (Eds.), Biological and behavioral determinants of language development. Hillsdale, NJ: Erlbaum.

Nelson, K. E. (2000). Methods for stimulating and measuring lexical and syntactic advances: Why Fiffins and lobsters can tag along with other recast friends. In L. Menn \& N. B. Ratner (Eds.), Methods for studying language production. Hillsdale, NJ. Erlbaum.

Nelson, K. (2006). A dynamic tricky mix theoretical perspective on educational interventions to support the acquisition of L1 and L2 spoken languages, sign languages, text, and art. Studies in Language Sciences, 5, 91-102.

Nelson, K. E., Welsh, J., Camarata, S., Heimann, \& Tjus, T. (2001). A rare event transactional dynamic model of tricky mix conditions contributing to language acquisition and varied communicative delays. In K. E. Nelson, A. Aksu-Koc, \& C. E. Johnson (Eds.), Children's language, Vol. 11. Hillsdale, NJ: Erlbaum.

Nelson, K. E., Craven, P. L., Xuan, Y., \& Arkenberg, M. E. (2004). Acquiring art, spoken language, sign language, text, and other symbolic systems: Developmental and evolutionary observations from a Dynamic Tricky Mix theoretical perspective. In J. M. Lucariello, J. A. Harris, R. Fivush, \& P. J. Bauer (Eds.), The development of the mediated mind. Sociocultural context and cognitive development (pp. 175-222). Mahwah, NJ: Lawrence Erlbaum Associates.

Nelson, K. \& Arkenberg, M. (2008). Language and reading development reflect dynamic mixes of learning conditions. Brain, behavior, and learning in language and reading disorders (pp. 315-348). New York: Guilford Press.

Newport, E.L. (1990). Maturational constraints on language learning. Cognitive Science, 14, 11-28.

Peltzer-Karpf, A. \& Zangl, R. (2001). Figure-ground segregation in visual and linguistic development: A Dynamic Systems Account. In K. E. Nelson, A. Aksu-Koc, \& C. E. Johnson (Eds.), Children's language, Vol. 11. Hillsdale, NJ: Erlbaum.

Repovs. G. \& Baddeley, A. (2006). The multi-component model of working memory: Explorations in experimental cognitive psychology. Neuroscience, 139, 5-21.

Roberts, R. \& Pennington, B. (1996). An interactive framework for examining prefrontal cognitive processes. Developmental Neuropsychology, 12(1), 105-126.

Ronnberg, J., Rudner, M., Foo, C., \& Lunner, T. (2008). Cognition counts: A working memory system for ease of language understanding (ELU). International Journal of Audiology, 47, 171-2177. 
Shallice, T. (2004). The fractionation of supervisory control. In M. Gazzaniga (Ed.), The cognitive neurosciences (3rd ed.) (pp. 1265-1277). Cambridge, MA: MIT Press.

Thelen, E. \& Smith, L. (1996). A dynamic systems approach to the development of cognition and action. Cambridge, MA: MIT Press.

Turner, M. L. \& Engle, R. W. (1989). Is working memory capacity task dependent? Journal of Memory and Language, 28, 127-154.

Correspondence: Keith E. Nelson, Department of Psychology, Pennsylvania State University, University Park, Pennsylvania, United States 